\title{
Divergência genética entre acessos de um banco de germoplasma de capim-elefante ${ }^{(1)}$
}

\author{
Aldo Shimoya( ${ }^{(2)}$, Cosme Damião $\mathrm{Cruz}^{(3)}$, Reinaldo de Paula Ferreira(4), \\ Antônio Vander Pereira ${ }^{(4)}$ e Pedro Crescencio Souza Carneiro ${ }^{(3)}$
}

Resumo - O objetivo deste trabalho foi estudar a divergência genética entre 99 acessos de capim-elefante (Pennisetum purpureum) do Banco de Germoplasma da Embrapa, em Coronel Pacheco, MG. O delineamento experimental foi o de blocos ao acaso, com cinco repetições de 99 tratamentos (97 genótipos e duas testemunhas). Foram avaliadas 17 características quantitativas. Utilizaram-se as análises multivariadas: distância de Mahalanobis, método de Tocher e variáveis canônicas. Pelo método de Tocher, 99 genótipos de capim-elefante foram agrupados em 18 grupos. Pela análise de variáveis canônicas, $81,80 \%$ da variância acumulada foi explicada pelas sete primeiras variáveis canônicas. Quanto à importância relativa das características avaliadas, diâmetro do colmo, largura da lâmina no meio da folha mediana adulta, largura da lâmina na base da folha mediana adulta, comprimento da arista, comprimento da espigueta, comprimento da folha mediana adulta e largura da folha-bandeira foram as que menos contribuíram; porém, não foram descartadas, uma vez que no novo agrupamento realizado após descarte de diâmetro do colmo houve alteração no número de grupos e na posição dos genótipos estabelecidos pelo método de Tocher. Foram indicados vários cruzamentos envolvendo a testemunha G98 e os genótipos promissores divergentes, visando ao aumento da variabilidade e a possibilidade de identificação de segregantes transgressivos.

Termos para indexação: Pennisetum purpureum, genótipos, distância genética, variabilidade genética, seleção, forrageiras.

\section{Genetic divergence among accessions of a germplasm bank of elephantgrass}

\begin{abstract}
The purpose of this work was to study the genetic divergence among 99 accessions of the active genebank of elephantgrass (Pennisetum purpureum) of Embrapa, in Coronel Pacheco, MG, Brazil. The experimental design used was randomized blocks, with 99 treatments ( 97 genotypes and two controls), with five replications. The evaluation involved 17 quantitative traits. The following multivariate analyses were used: distance of Mahalanobis, Tocher's method, and canonical variables. By Tocher's method the 99 genotypes were grouped in 18 clusters. According to the analysis of canonical variables, $81.80 \%$ of the accumulated variance were explained by the first seven canonical variables. The relative importance of the characteristics was determined and those that contributed less to the genetic divergence were: diameter of the stem, width of the half blade of the medium complete leaf, width of the lower blade of the medium complete leaf, length of the awn, length of the spikelet, length of the medium complete leaf and width of the flag leaf. One test eliminating diameter of the stem altered the number of groups and the position of the genotypes established by the method of Tocher, as an illustration of the use of this technique. Several crossings were indicated, involving the control G98 and the promising divergent genotypes to attain increases in variability and the possibility of identification of transgressive segregants.
\end{abstract}

Index terms: Pennisetum purpureum, genotypes, genetic distance, genetic variability, selection, forage.

\footnotetext{
(1) Aceito para publicação em 17 de dezembro de 2001. Extraído da tese de doutorado, apresentada pelo primeiro autor à Universidade Federal de Viçosa (UFV), Viçosa, MG

(2) Pesagro-Rio, Estacão Experimental de Campos, Caixa Postal 114331, CEP 28080-000 Campos dos Goytacazes, RJ. E-mail: aldos@censanet.com.br
}

(3) UFV, Dep. de Biologia Geral, CEP 36571-000 Viçosa, MG

(4) Embrapa-Centro Nacional de Pesquisa de Gado de Leite, Rua Eugênio do Nascimento 610, CEP 36038-330 Juiz de Fora, MG 


\section{Introdução}

O conhecimento do grau de divergência genética é de grande importância em programas de melhoramento que envolvem hibridações, por fornecer parâmetros para a identificação de progenitores que, quando cruzados, possibilitam maior efeito heterótico na progênie e maior probabilidade de recuperar genótipos superiores nas gerações segregantes (Jatasra \& Paroda, 1983; Cruz et al., 1994; Santos et al., 1994; Samal \& Jagadev, 1996; Pandey \& Dobhal, 1997).

A análise de divergência genética tem sido usada pelos melhoristas, há várias décadas, e os auxilia na classificação de genótipos em grupos e na escolha de progenitores com características desejáveis para hibridação. É enfatizada em trabalhos com diferentes culturas (Julfiquar et al., 1985; Jaylal \& Haider, 1994; Ali et al., 1995; Dwivedi \& Mitra, 1996). Segundo Wilches (1983), o agrupamento e a avaliação de material genético por meio da diversidade genética, com base em evidências científicas, são importantes para o conhecimento da evolução da espécie e para a localização e o intercâmbio de recursos genéticos.

A divergência genética entre populações pode ser avaliada de várias maneiras. Miranda et al. (1988) mostram duas maneiras básicas: uma de natureza quantitativa, e a outra de natureza preditiva. Entre os métodos de natureza quantitativa, ou da heterose manifestada nos híbridos, citam-se os de análises dialélicas, para os quais é necessária a avaliação de $\mathrm{p}$ progenitores e de todas as suas combinações híbridas $[\mathrm{p}(\mathrm{p}-1) / 2]$. Quando o valor de $\mathrm{p}$ é elevado, a obtenção do material experimental pode ser impraticável. Entre os métodos preditivos de heteroses, Miranda et al. (1988) citam os que relacionam a divergência das características dos progenitores com o desempenho dos híbridos. Os métodos preditivos são os que levam em consideração, entre outras, as características agronômicas, fisiológicas, genéticas e morfológicas, apresentadas pelos progenitores na determinação da divergência (Rao et al., 1981; Singh et al., 1981; Cruz, 1990; Carvalho et al., 1995), que é, geralmente, quantificada pela estatística multivariada, distância euclidiana ou distância generalizada de Mahalanobis.
O objetivo deste trabalho foi estudar a divergência genética de 99 acessos de capim-elefante (Pennisetum purpureum).

\section{Material e Métodos}

Foi estudado o grau de divergência genética entre os genótipos de capim-elefante a partir de dados provenientes de um experimento realizado na Embrapa-Centro Nacional de Pesquisa de Gado de Leite (CNPGL), Município de Coronel Pacheco, MG.

O experimento foi constituído de 99 acessos do Banco Ativo de Germoplasma de Capim-Elefante (BAGCE), que foi implantado num Latossolo Vermelho-Amarelo, de meia encosta, representativo da região. A aplicação de calcário e de fertilizantes foi feita com base nas análises química e granulométrica de amostras de solo $(0-20 \mathrm{~cm})$ e no requerimento nutricional da espécie. O plantio foi realizado com mudas, em sulcos com $0,20 \mathrm{~m}$ de profundidade. Após a fase de estabelecimento dos acessos, cerca de 90 dias após o plantio, as plantas de todos os tratamentos foram cortadas a $0,30 \mathrm{~m}$ da superfície do solo, procedendo-se, assim, ao início da fase de coleta de dados.

Entre os acessos avaliados, estão cultivares melhoradas, clones e introduções de várias regiões do país e do exterior, que no presente trabalho são referidos como acessos ou genótipos. A cultivar Pioneiro, desenvolvida pelo programa de melhoramento de capim-elefante da EmbrapaCNPGL, foi utilizada como uma das testemunhas. Essa cultivar, registrada no BAGCE como CNPGL 91 F 27-1, correspondente ao número do acesso G98, foi obtida pelo cruzamento entre as cultivares Três Rios e Mercker Santa Rita, progenitores masculino e feminino, respectivamente. A outra testemunha utilizada, registrada no BAGCE como CNPGL 91 F 27-5, correspondente ao número do acesso G99, foi obtida pelo mesmo cruzamento. Os acessos G98 e G99 foram considerados como testemunhas, por apresentarem características superiores, tais como produção de matéria seca, alta relação folha/colmo, mais altos teores de proteína e melhor digestibilidade, em comparação com outras cultivares disponíveis.

O delineamento experimental utilizado foi o de blocos ao acaso, com cinco repetições. Cada parcela foi constituída de uma linha de 2,00 m, e o espaçamento entre as linhas de diferentes parcelas foi de $2,50 \mathrm{~m}$. As avaliações foram realizadas na área útil $\left(4,25 \mathrm{~m}^{2}\right)$ de cada parcela, considerando bordadura de $0,15 \mathrm{~m}$ nas extremidades de cada linha.

Os tratamentos foram avaliados por meio de cinco amostragens, logo após a rebrota e durante as fases vegetativa e reprodutiva. Foram avaliadas 17 características: 1) diâmetro da ráquis; 2) comprimento da folha-ban- 
deira; 3 ) largura da folha-bandeira; 4) diâmetro da inflorescência; 5) comprimento da inflorescência; 6) comprimento da lígula; 7) comprimento da arista; 8) comprimento da espigueta; 9) número de flósculo por espigueta; 10) diâmetro do colmo; 11) altura da planta; 12) comprimento da folha mediana adulta; 13) comprimento do entrenó da folha mediana adulta; 14) largura da lâmina na base da folha mediana adulta; 15) largura da lâmina no meio da folha mediana adulta; 16) ângulo de inserção da folha mediana adulta; 17) comprimento da bainha da folha mediana adulta.

Os dados obtidos foram submetidos à análise de variância, a fim de verificar a existência de variabilidade genética entre os acessos e as médias comparadas pelo teste de Scott-Knott a 5\% de probabilidade (Scott \& Knott, 1974).

Foi utilizada a análise multivariada, aplicando-se as técnicas de agrupamento e de variáveis canônicas. Na técnica de agrupamento, foi utilizada a distância generalizada de Mahalanobis como medida de dissimilaridade, e na delimitação dos grupos, o método de otimização de Tocher, citado por Rao (1952). Na análise de variáveis canônicas, a divergência genética foi evidenciada por meio de gráficos cartesianos de dispersão dos escores, sendo os eixos representados pelas primeiras variáveis canônicas. Foi estudada também a importância relativa de cada variável canônica (Cruz \& Regazzi, 1997) e a importância relativa das características na predição da divergência genética. As análises estatísticas foram processadas por programa computacional GENES (Cruz, 1997).

\section{Resultados e Discussão}

$\mathrm{O}$ efeito dos tratamentos apresentou diferenças significativas $(\mathrm{P}<0,01) \mathrm{em}$ todas as características avaliadas. Este fato é um indicativo da condição favorável à realização do melhoramento para essas características, o que torna possível a identificação de materiais genéticos superiores e a obtenção de ganhos consideráveis com sua introdução em um programa de melhoramento. Entre as características avaliadas, comprimento e largura da folha, diâmetro do caule, comprimento do internódio, altura da planta e ângulo de inserção da folha têm sido correlacionadas com a produção e qualidade da matéria seca (Tcacenco, 1992; Diz \& Schank, 1995; Shimoya, 2000), indicando suas importâncias como critério de seleção nos programas de melhoramento.

A identificação dos genitores foi feita com base em características vegetativas e reprodutivas impor- tantes, como, por exemplo, a medida direta e indireta da produtividade, e a qualidade da forrageira (Tabela 1).

A partir da distância generalizada de Mahalanobis, utilizando 17 características quantitativas em 99 genótipos de capim-elefante, os genótipos G40 e G86 foram os mais dissimilares, apresentando a maior distância (365,48), e os genótipos G72 e G76 foram os mais similares, com a menor distância $(5,71)$. Os genótipos G46, G56, G40 e G85 foram os que apresentaram as maiores distâncias e os genótipos G23 e G88, as menores, em relação às testemunhas G98 e G99, simultaneamente. As distâncias das testemunhas em relação aos cinco genótipos mais similares foram próximas, indicando, talvez, que estas testemunhas são pouco divergentes, assim como os genótipos a elas relacionados.

Com base na magnitude relativa de valores de $\mathrm{D}^{2}$, os 99 genótipos foram agrupados em 18 grupos (Tabela 2). O mesmo procedimento inicial na formação dos 18 grupos, a partir dos 99 genótipos, foi realizado no grupo 1, composto de 44 genótipos, que foram reagrupados em 14 subgrupos. Já era esperado que os genótipos $\mathrm{G} 72$ e G76 pertencessem ao mesmo subgrupo, por serem os mais similares. Os genótipos G40 e G86, considerados os mais dissimilares, foram agrupados, respectivamente, nos grupos 2 e 12 . As testemunhas G98 e G99 ficaram no mesmo grupo (grupo 5), resultado este esperado, uma vez que suas distâncias relativas aos cinco genótipos mais similares foram consideradas próximas.

A importância relativa das duas primeiras variáveis canônicas explicou somente $50,02 \%$ da variância total (Tabela 3). Conforme Cruz \& Regazzi (1997), a viabilidade do uso dessa técnica para identificação de genótipos similares em gráficos bi ou tridimensionais depende da concentração da variância total disponível acima de $80 \%$ entre as primeiras variáveis canônicas. Como isso não foi alcançado, a análise foi complementada com a dispersão gráfica em relação à terceira, quarta, quinta, sexta e sétima variável canônica, perfazendo 81,80\%.

Os genótipos G72 e G76 apresentaram posições bastante similares, ao passo que os genótipos G40 e G86 foram os mais dissimilares (Figura 1). Esses genótipos mostraram comportamento semelhante em relação às demais variáveis canônicas, o que ratifica 
Tabela 1. Médias das características: diâmetro da ráquis (DRA), comprimento da folha-bandeira(CFB), largura da folha-bandeira (LFB), diâmetro da inflorescência (DIN), comprimento da inflorescência (CIN), comprimento da lígula (CLI), comprimento da arista (CAR), comprimento da espigueta (CES), número de flósculo por espigueta (NFL), diâmetro do colmo (DCO), altura da planta (APL), comprimento da folha mediana adulta (CFO), comprimento do entrenó da folha mediana adulta (CEN), largura da lâmina na base da folha mediana adulta (LBA), largura da lâmina no meio da folha mediana adulta (LME), ângulo de inserção da folha mediana adulta (AIN) e comprimento da bainha da folha mediana adulta (CBA), obtidas de genótipos de capim-elefante ${ }^{(1)}$.

\begin{tabular}{|c|c|c|c|c|c|c|c|c|c|c|c|c|c|c|c|c|c|}
\hline Genótipos $^{(2)}$ & $\begin{array}{l}\text { DRA } \\
(\mathrm{cm})\end{array}$ & $\begin{array}{l}\text { CFB } \\
(\mathrm{cm})\end{array}$ & $\begin{array}{l}\text { LFB } \\
(\mathrm{cm})\end{array}$ & $\begin{array}{l}\text { DIN } \\
(\mathrm{cm})\end{array}$ & $\begin{array}{l}\mathrm{CIN} \\
(\mathrm{cm})\end{array}$ & $\begin{array}{l}\text { CLI } \\
(\mathrm{cm})\end{array}$ & $\begin{array}{l}\text { CAR } \\
(\mathrm{cm})\end{array}$ & $\begin{array}{l}\text { CES } \\
(\mathrm{cm})\end{array}$ & NFL & $\begin{array}{l}\text { DCO } \\
(\mathrm{cm})\end{array}$ & $\begin{array}{l}\text { APL } \\
(\mathrm{m})\end{array}$ & $\begin{array}{l}\mathrm{CFO} \\
(\mathrm{cm})\end{array}$ & $\begin{array}{l}\text { CEN } \\
(\mathrm{cm})\end{array}$ & $\begin{array}{l}\text { LBA } \\
(\mathrm{cm})\end{array}$ & $\begin{array}{l}\text { LME } \\
(\mathrm{cm})\end{array}$ & AIN & $\begin{array}{l}\text { CBA } \\
(\mathrm{cm})\end{array}$ \\
\hline G6 & $0,242 \mathrm{c}$ & $15,72 \mathrm{e}$ & $1,38 \mathrm{~d}$ & $1,600 \mathrm{~b}$ & $26,70 \mathrm{~b}$ & $0,376 a$ & $2,278 \mathrm{a}$ & $0,706 \mathrm{c}$ & $2,4 a$ & $1,266 \mathrm{a}$ & $4,230 \mathrm{a}$ & $102,0 \mathrm{~d}$ & $15,7 \mathrm{~b}$ & $2,92 \mathrm{a}$ & $3,44 \mathrm{c}$ & $29,0 \mathrm{~d}$ & $19,8 \mathrm{c}$ \\
\hline G10 & $0,182 \mathrm{e}$ & $8,50 \mathrm{f}$ & $0,66 f$ & $1,426 c$ & $26,04 b$ & $0,250 \mathrm{c}$ & $1,948 \mathrm{a}$ & $0,614 \mathrm{c}$ & $3,0 \mathrm{a}$ & $0,914 \mathrm{c}$ & $3,080 \mathrm{~d}$ & $100,0 \mathrm{~d}$ & $11,8 \mathrm{~d}$ & $1,12 \mathrm{~d}$ & $3,16 \mathrm{~d}$ & $20,0 \mathrm{~d}$ & $20,1 \mathrm{c}$ \\
\hline G13 & $0,214 \mathrm{~d}$ & $11,70 \mathrm{e}$ & $1,06 \mathrm{e}$ & $1,702 \mathrm{~b}$ & $23,98 \mathrm{c}$ & $0,332 b$ & $1,548 \mathrm{c}$ & $0,574 \mathrm{~d}$ & $3,0 \mathrm{a}$ & $1,198 \mathrm{a}$ & $4,310 \mathrm{a}$ & $105,8 \mathrm{c}$ & $14,9 b$ & $2,40 \mathrm{~b}$ & $4,06 \mathrm{~b}$ & $34,0 \mathrm{c}$ & $21,9 b$ \\
\hline G18 & $0,204 d$ & $10,48 \mathrm{f}$ & $0,80 \mathrm{f}$ & $1,510 \mathrm{~b}$ & $26,30 \mathrm{~b}$ & $0,254 \mathrm{c}$ & $1,598 \mathrm{~b}$ & $0,764 \mathrm{~b}$ & $2,8 \mathrm{a}$ & $0,828 \mathrm{c}$ & $3,350 \mathrm{c}$ & $111,6 b$ & $14,1 \mathrm{c}$ & $2,04 \mathrm{c}$ & $3,40 \mathrm{c}$ & $34,0 \mathrm{c}$ & $17,3 \mathrm{e}$ \\
\hline G20 & $0,222 \mathrm{~d}$ & $9,90 \mathrm{f}$ & $0,72 \mathrm{f}$ & $1,720 \mathrm{~b}$ & $25,50 \mathrm{~b}$ & $0,428 \mathrm{a}$ & $1,762 \mathrm{~b}$ & $0,566 \mathrm{~d}$ & $3,0 \mathrm{a}$ & $1,026 \mathrm{~b}$ & $3,910 \mathrm{~b}$ & $105,4 \mathrm{c}$ & $14,0 \mathrm{c}$ & $2,34 \mathrm{~b}$ & $3,42 \mathrm{c}$ & $41,0 \mathrm{c}$ & $18,0 \mathrm{~d}$ \\
\hline G36 & $0,248 \mathrm{c}$ & $20,10 \mathrm{~d}$ & $1,30 \mathrm{~d}$ & $2,144 \mathrm{a}$ & $23,90 \mathrm{c}$ & $0,342 b$ & $1,314 \mathrm{~d}$ & $0,534 \mathrm{~d}$ & $1,6 \mathrm{c}$ & $0,464 \mathrm{e}$ & $3,080 \mathrm{~d}$ & $103,6 \mathrm{c}$ & $11,4 \mathrm{~d}$ & $1,58 \mathrm{~d}$ & $2,90 \mathrm{~d}$ & $31,0 \mathrm{~d}$ & $21,0 \mathrm{c}$ \\
\hline G43 & $0,264 b$ & $11,42 \mathrm{e}$ & $0,98 \mathrm{e}$ & $1,458 \mathrm{c}$ & $30,90 \mathrm{a}$ & $0,336 \mathrm{~b}$ & $1,550 \mathrm{c}$ & $0,610 \mathrm{c}$ & $2,4 \mathrm{a}$ & $0,960 \mathrm{~b}$ & $3,590 \mathrm{c}$ & $99,2 \mathrm{~d}$ & $14,4 \mathrm{c}$ & $2,04 \mathrm{c}$ & $3,76 \mathrm{c}$ & $42,0 \mathrm{c}$ & $18,3 \mathrm{~d}$ \\
\hline G50 & $0,190 \mathrm{e}$ & $11,84 \mathrm{e}$ & $1,00 \mathrm{e}$ & $1,322 \mathrm{c}$ & $19,38 \mathrm{~d}$ & $0,214 d$ & $2,062 \mathrm{a}$ & $0,664 c$ & $3,0 \mathrm{a}$ & $0,792 \mathrm{c}$ & $3,540 \mathrm{c}$ & $111,8 \mathrm{~b}$ & $12,4 \mathrm{~d}$ & $2,48 \mathrm{~b}$ & $3,96 \mathrm{~b}$ & $42,0 \mathrm{c}$ & $20,2 \mathrm{c}$ \\
\hline G53 & $0,274 b$ & $13,80 \mathrm{e}$ & $1,14 \mathrm{e}$ & $1,974 \mathrm{a}$ & $23,94 \mathrm{c}$ & $0,194 d$ & $1,570 \mathrm{c}$ & $0,616 \mathrm{c}$ & $1,2 \mathrm{c}$ & $0,912 \mathrm{c}$ & $3,170 \mathrm{~d}$ & $85,6 \mathrm{e}$ & $15,1 b$ & $1,98 \mathrm{c}$ & $3,30 \mathrm{~d}$ & $41,0 \mathrm{c}$ & $22,3 \mathrm{~b}$ \\
\hline G56 & $0,166 \mathrm{f}$ & $18,74 \mathrm{~d}$ & $1,30 \mathrm{~d}$ & $1,270 \mathrm{~d}$ & $17,30 \mathrm{~d}$ & $0,278 \mathrm{c}$ & $1,402 \mathrm{c}$ & $0,530 \mathrm{~d}$ & $1,2 \mathrm{c}$ & $0,422 \mathrm{e}$ & $1,740 \mathrm{~g}$ & $73,4 \mathrm{f}$ & $9,1 \mathrm{~d}$ & $1,42 \mathrm{~d}$ & $3,06 \mathrm{~d}$ & $26,0 \mathrm{~d}$ & $12,3 \mathrm{~g}$ \\
\hline G59 & $0,188 \mathrm{e}$ & $19,90 \mathrm{~d}$ & $1,34 \mathrm{~d}$ & $1,464 \mathrm{c}$ & $22,10 \mathrm{c}$ & $0,170 \mathrm{~d}$ & $1,782 b$ & $0,490 \mathrm{~d}$ & $1,8 \mathrm{~b}$ & $0,978 b$ & $3,090 \mathrm{~d}$ & $107,4 \mathrm{c}$ & $10,6 \mathrm{~d}$ & $1,38 \mathrm{~d}$ & $3,14 \mathrm{~d}$ & $31,0 \mathrm{~d}$ & $20,2 \mathrm{c}$ \\
\hline G60 & $0,256 \mathrm{c}$ & $20,50 \mathrm{~d}$ & $1,56 \mathrm{c}$ & $1,572 \mathrm{~b}$ & $31,30 \mathrm{a}$ & $0,196 \mathrm{~d}$ & $2,012 \mathrm{a}$ & $0,604 \mathrm{c}$ & $2,4 \mathrm{a}$ & $0,846 \mathrm{c}$ & $3,720 \mathrm{c}$ & $115,4 \mathrm{~b}$ & $11,7 \mathrm{~d}$ & $1,76 \mathrm{c}$ & $3,66 \mathrm{c}$ & $29,0 \mathrm{~d}$ & $23,0 \mathrm{~b}$ \\
\hline G64 & $0,166 \mathrm{f}$ & $3,62 \mathrm{~g}$ & $0,48 \mathrm{~g}$ & $1,650 \mathrm{~b}$ & $19,10 \mathrm{~d}$ & $0,260 \mathrm{c}$ & $1,510 \mathrm{c}$ & $0,550 \mathrm{~d}$ & $2,4 \mathrm{a}$ & $0,718 \mathrm{c}$ & $3,400 \mathrm{c}$ & $92,2 \mathrm{e}$ & $17,4 \mathrm{a}$ & $2,32 b$ & $3,28 \mathrm{~d}$ & $65,0 \mathrm{a}$ & $14,0 \mathrm{f}$ \\
\hline G65 & $0,232 \mathrm{c}$ & $31,20 \mathrm{~b}$ & $1,74 \mathrm{~b}$ & $1,250 \mathrm{~d}$ & $29,60 \mathrm{a}$ & $0,260 \mathrm{c}$ & $1,418 \mathrm{c}$ & $0,622 \mathrm{c}$ & $2,8 \mathrm{a}$ & $1,094 \mathrm{~b}$ & $3,260 \mathrm{~d}$ & $115,4 \mathrm{~b}$ & $14,0 \mathrm{c}$ & $2,18 \mathrm{~b}$ & $4,36 \mathrm{~b}$ & $18,0 \mathrm{~d}$ & $20,4 \mathrm{c}$ \\
\hline G71 & $0,242 \mathrm{c}$ & $7,62 \mathrm{f}$ & $0,60 \mathrm{~g}$ & $1,666 b$ & $24,84 b$ & $0,292 \mathrm{~b}$ & $1,836 \mathrm{~b}$ & $0,640 \mathrm{c}$ & $2,6 \mathrm{a}$ & $0,714 \mathrm{c}$ & $3,090 \mathrm{~d}$ & $98,0 \mathrm{~d}$ & $19,5 \mathrm{a}$ & $2,00 \mathrm{c}$ & $3,30 \mathrm{~d}$ & $57,0 \mathrm{~b}$ & $14,5 \mathrm{f}$ \\
\hline G74 & $0,206 \mathrm{~d}$ & $30,90 \mathrm{~b}$ & $2,04 \mathrm{a}$ & $1,308 \mathrm{c}$ & $31,52 \mathrm{a}$ & $0,264 \mathrm{c}$ & $1,784 \mathrm{~b}$ & $0,640 \mathrm{c}$ & $2,0 \mathrm{~b}$ & $1,324 \mathrm{a}$ & $3,566 \mathrm{c}$ & $115,6 \mathrm{~b}$ & $13,1 \mathrm{c}$ & $2,86 \mathrm{a}$ & $4,80 \mathrm{a}$ & $35,0 \mathrm{c}$ & $20,6 \mathrm{c}$ \\
\hline G86 & $0,300 \mathrm{~b}$ & $22,28 \mathrm{~d}$ & $1,76 \mathrm{~b}$ & $2,022 \mathrm{a}$ & $30,68 \mathrm{a}$ & $0,272 \mathrm{c}$ & $1,488 \mathrm{c}$ & $0,518 \mathrm{~d}$ & $2,2 \mathrm{~b}$ & $1,344 \mathrm{a}$ & $4,100 \mathrm{~b}$ & $123,6 \mathrm{a}$ & $11,5 \mathrm{~d}$ & $3,06 \mathrm{a}$ & $4,60 \mathrm{a}$ & $35,0 \mathrm{c}$ & $20,5 \mathrm{c}$ \\
\hline G98 & $0,196 \mathrm{e}$ & $23,44 d$ & $1,34 \mathrm{~d}$ & $1,622 b$ & $26,08 \mathrm{~b}$ & $0,358 \mathrm{a}$ & $1,450 \mathrm{c}$ & $0,556 \mathrm{~d}$ & $2,2 b$ & $1,056 \mathrm{~b}$ & $3,100 \mathrm{~d}$ & $103,6 \mathrm{c}$ & $14,8 b$ & $2,52 \mathrm{~b}$ & $3,22 \mathrm{~d}$ & $63,0 \mathrm{a}$ & $18,9 \mathrm{~d}$ \\
\hline
\end{tabular}


as informações contidas nas duas primeiras variáveis canônicas e confirma os resultados anteriormente discutidos na análise de agrupamento de Tocher. As duas primeiras variáveis canônicas explicaram cerca de $50 \%$ da variância total, que apenas os grupos d (13 genótipos), e (seis genótipos) e f (dois genótipos) foram agrupados de modo mais perceptível e que à medida que se fez a complementação gráfica, até atingir mais de $80 \%$ da variância total (Figura 1A), outros grupos tornaram-se perceptíveis, como o grupo a (44 genótipos) (Figura 1B); o grupo c (12 genótipos) (Figura 1C); o grupo g (dois genótipos) (Figura 1D); os grupos f(dois genótipos) e b (oito genótipos) (Figura 1E); e o grupo h (dois genótipos) (Figura 1F). Este fato pode ter ocorrido em virtude de terem sido considerados dois eixos cartesianos para a dispersão gráfica dos genótipos, a saber: um eixo fixo, representado pela primeira variável canônica, e o outro, não-fixo, representado pelas seis variáveis canônicas subseqüentes, que fo-

Tabela 2. Grupos de similaridade genética entre 99 genótipos de capim-elefante, estabelecidos pelo método de Tocher, a partir da distância generalizada de Mahalanobis.

\begin{tabular}{ccl}
\hline Grupos & Subgrupos & \multicolumn{1}{c}{ Genótipos } \\
\hline 1 & $\mathrm{a}$ & $72,76,69,51,32,31 \mathrm{e} 30$ \\
1 & $\mathrm{~b}$ & $22,25,28,96,44,92,83,15 \mathrm{e} 88$ \\
1 & $\mathrm{c}$ & $9,29,11,14,24,27,54 \mathrm{e} 75$ \\
1 & $\mathrm{~d}$ & $64 \mathrm{e} 87$ \\
1 & $\mathrm{e}$ & $91,94,95 \mathrm{e} 23$ \\
1 & $\mathrm{f}$ & $33,70 \mathrm{e} 35$ \\
1 & $\mathrm{~g}$ & $57 \mathrm{e} 77$ \\
1 & $\mathrm{~h}$ & $42 \mathrm{e} 78$ \\
1 & $\mathrm{i}$ & $17 \mathrm{e} 34$ \\
1 & $\mathrm{j}$ & 48 \\
1 & $\mathrm{k}$ & 1 \\
1 & 1 & 45 \\
1 & $\mathrm{~m}$ & 21 \\
1 & $\mathrm{n}$ & 7 \\
2 & - & $40,85,39,84,82,81,67 \mathrm{e} 38$ \\
3 & - & $66,74,52,65,61,55,58,68,60,63,89$ e 37 \\
4 & - & $8,50,5,4,13,12,18,20,43,19,10,59 \mathrm{e} 3$ \\
5 & - & $26,90,99,93,98 \mathrm{e} 16$ \\
6 & - & $2 \mathrm{e} 6$ \\
7 & - & $47 \mathrm{e} 49$ \\
8 & - & $36 \mathrm{e} 53$ \\
9 & - & 73 \\
10 & - & 62 \\
11 & - & 71 \\
12 & - & 86 \\
13 & - & 97 \\
14 & - & 79 \\
15 & - & 41 \\
16 & - & 80 \\
17 & - & 56 \\
18 & - & 46 \\
\hline & &
\end{tabular}

ram utilizadas para a complementação. A posição dos genótipos nos gráficos de dispersão foi determinada por meio das estimativas dos escores obtidos a partir das variáveis canônicas. Quando foi realizada a complementação de uma variável canônica no eixo não-fixo ocorreu uma mudança de escores, e, conseqüentemente, da posição dos genótipos, o que tornou perceptível a formação de grupos anteriormente não vistos. Os grupos formados por um genótipo são perceptíveis em todos os gráficos.

Para avaliar a importância relativa das características na divergência genética, foram identificadas nas últimas variáveis canônicas, as menos importantes como sendo aquelas cujos coeficientes de ponderação são de maior magnitude, em valor absoluto (Cruz \& Regazzi, 1997). As características de menor importância foram, em ordem de descarte, diâmetro do colmo, na $\mathrm{VC}_{17}$; largura da lâmina no meio da folha mediana adulta, na $\mathrm{VC}_{16}$; largura da lâmina na base da folha mediana adulta, na $\mathrm{VC}_{15}$; comprimento da arista, na $\mathrm{VC}_{14}$; comprimento da espigueta, na $\mathrm{VC}_{13}$; comprimento da folha mediana adulta, na $\mathrm{VC}_{12}$; e largura da folha-bandeira, na $\mathrm{VC}_{10}$ (Tabela 3). A possibilidade de descarte das características que contribuem pouco para a discriminação do material genético avaliado é importante, pois permite a redução da mão-de-obra, do tempo e do custo despendidos na experimentação (Cruz, 1990; Cruz \& Regazzi, 1997). Quando se descartou a característica de menor importância relativa (diâmetro do colmo) pelo método das variáveis canônicas, conforme Cruz \& Regazzi (1997), o número de agrupamentos, estabelecidos pelo método de otimização de Tocher, passou de 18 para 19 e a posição dos genótipos também foi modificada (Tabela 4). Isso indica que apesar de essa característica apresentar o maior coeficiente de ponderação no componente de menor variância, explicando uma fração mínima da variância total, ela não pode ser descartada. O procedimento de Cruz \& Regazzi (1997) baseia-se no critério de descarte de características redundantes ou invariantes, mas este método não foi eficaz quando se considerou a importância da variável com base na alteração do padrão de agrupamento estabelecido pelo método de Tocher, como ocorreu no presente estudo.

A variabilidade genética existente entre os 99 acessos de capim-elefante do BAGCE permitiu indicar 
Tabela 3. Estimativas das variâncias (autovalores $\left.\lambda_{\mathrm{j}}\right)$, das porcentagens da variância acumulada $(\lambda \%)$, explicadas pelas variáveis canônicas $\left(\mathrm{VC}_{\mathrm{i}}\right)$, e dos respectivos coeficientes que expressam a importância relativa das características quantitativas: diâmetro da ráquis (DRA), comprimento da folha-bandeira (CFB), largura da folha-bandeira (LFB), diâmetro da inflorescência (DIN), comprimento da inflorescência (CIN), comprimento da lígula (CLI), comprimento da arista (CAR), comprimento da espigueta (CES), número de flósculos por espigueta (NFL), diâmetro do colmo (DCO), altura da planta (APL), comprimento da folha mediana adulta (CFO), comprimento do entrenó da folha mediana adulta (CEN), largura da lâmina na base da folha mediana adulta (LBA), largura da lâmina no meio da folha mediana adulta (LME), ângulo de inserção da folha mediana adulta (AIN) e comprimento da bainha da folha mediana adulta (CBA), avaliadas em 99 genótipos de capim-elefante.

\begin{tabular}{|c|c|c|c|c|c|c|c|c|c|c|c|c|c|c|c|c|c|c|c|}
\hline \multirow[t]{2}{*}{$\mathrm{VC}_{\mathrm{i}}$} & \multicolumn{2}{|c|}{ Variância } & \multicolumn{17}{|c|}{ Importância relativa das características ${ }^{(1)}$} \\
\hline & $\lambda_{j}$ & \%) & RA & CFB & LFB & DIN & CIN & CLI & CAR & $\mathrm{Cl}$ & NFL & 0 & APL & FO & CEN & LBA & LME & AIN & A \\
\hline$E_{1}$ & 5,283 & 4048 & 492 & 0193 & 0,3278 & 0,1593 & 0,4241 & $-0,1109$ & 0,0635 & 3308 & 1636 & 0,3 & 2270 & 3305 & $-0,1576$ & 0,3000 & 0,4090 & $-0,1944$ & 3926 \\
\hline $\mathrm{vC}$ & & & 848 & 41 & 88 & $-0,38$ & 0587 & 4 & & & & & & 76 & & & & & \\
\hline $\mathrm{VC}_{3}$ & 940 & , 1958 & 1064 & $-0,2763$ & $-0,0306$ & 1519 & $-0,0209$ & 2731 & 46 & 50 & 45 & & & 66 & 21 & 22 & 30 & 45 & \\
\hline $\mathrm{VC}_{4}$ & 479 & 2624 & 827 & $-0,0963$ & 4322 & 1085 & 2579 & 0,2296 & $-0,0284$ & 0061 &, 0800 & $-0,0$ & 669 & $-0,14$ & 71 & 4510 & $-0,1981$ & 0,1208 & -0, \\
\hline $\mathrm{VC}_{5}$ & 839 & 2,6739 & 789 & 0,0611 & $-0,0253$ & $-0,0214$ & $-0,1306$ & 0,6270 & $-0,2927$ &, 0299 &, 1594 & ,03 & 1411 & ,5111 & $-0,2759$ & 0,1538 & $-0,0948$ &, 04 & \\
\hline $\mathrm{VC}_{6}$ & 1,9845 & 77,5983 & 470 & 1495 & 0,1446 & 0,1054 & $-0,4884$ & $-0,4898$ & $-0,0345$ & $-0,2169$ & $-0,2529$ & 0,0 & 65 & $-0,0148$ & 2903 & 3055 & 0,0691 & 0,1968 & \\
\hline $\mathrm{VC}$ & 935 & 8012 & 61 & 34 & 3345 & 4757 & 514 & 0984 & $-0,1$ & $-0,1724$ & 714 & & & 09 & 10 & 557 & 99 & 49 & \\
\hline $\mathrm{VC}_{8}$ & 1,4534 & 85,4069 & $-0,1535$ & 4156 & $-0,2942$ & 0,4664 & $-0,0450$ & 0,1978 & 0,4014 & 0,3118 & 0,2928 & $-0,0046$ & -0 , & $-0,1136$ & 0,0968 & 0239 & 0,0877 & 0,4125 & \\
\hline $\mathrm{VC}_{9}$ & 2169 & 4204 & 435 & $-0,0512$ & 0,0958 & $-0,5679$ & $-0,0614$ & $-0,1983$ & 0,461 & 260 & 83 & & & $-0,3$ & 0871 & 2132 & $-0,4036$ & $-0,1$ & \\
\hline $\mathrm{VC}_{10}$ & 0,9984 & 90,9039 & $-0,2119$ & $-0,6271$ & 0,7701 & $-0,1361$ & $-0,0371$ & 0,1214 & 0,1997 & $-0,0484$ & $-0,0639$ & ,2160 & $-0,1402$ & 0,1348 & $-0,0337$ & $-0,3160$ & 0,1479 & 0,4653 & 0837 \\
\hline $\mathrm{VC}_{11}$ & 0,8197 & 92,9379 & 335 & $-0,1073$ & 0,0260 & 0,0108 & $-0,5621$ & 0,2825 & 0,1352 & 61 & 9993 & & $-0,1$ & 0,0 & 0,2200 & $-0,3051$ & 0,7067 & $-0,4545$ & \\
\hline $\mathrm{VC}_{12}$ & 0,7215 & 94,7282 & 3684 & 0,1742 & $-0,1301$ & 0,0141 & $-0,3597$ & 0,0479 & 0,0741 & 0,1866 & $-0,1736$ & $-0,05$ & & 0,6224 & $-0,2739$ & $-0,3927$ & $-0,1542$ & 0,3102 & $-0,33$ \\
\hline $\mathrm{VC}_{13}$ & 0,635 & 96,3054 & 0075 & $-0,0730$ & $-0,0709$ & 0079 & 0,1221 & 0740 & $-0,1710$ & 80 &, 5269 & & & 98 & $-0,0321$ & 2847 & $-0,2028$ & 0,0473 & \\
\hline $\mathrm{VC}_{14}$ & 0,4429 & 91,4045 & 9930 & 0,0037 & $-0,1231$ & $-0,0950$ & $-0,0060$ & $-0,1817$ & $-0,5555$ & 0,2714 & 0,4826 & 0,2337 & $-0,1607$ & $-0,1728$ & $-0,2484$ & $-0,0477$ & 0,2893 & 0,2499 & $-0,0070$ \\
\hline $\mathrm{VC}$ & 1072 & 98,41 & & $-0,0461$ & $-0,0105$ & $-0,1899$ & $-0,0186$ & & & $-0,2533$ & 28 & & & & $-0,4691$ & & & & \\
\hline $\mathrm{VC}_{16}$ & 0,3711 & 99,3359 & 9984 & 0,0977 & $-0,3103$ & $-0,1011$ & 0,2037 & $-0,0640$ & 0,1757 & $-0,0213$ & $-0,4724$ & $-0,0540$ & 0,1954 & $-0,3340$ & $-0,1155$ & $-0,4101$ & 0,6847 & 0,2054 & $-0,0073$ \\
\hline $\mathrm{VC}_{17}$ & 0,2676 & , ,0000 & 1226 & 0548 & $-0,2636$ & $-0,0700$ & $-0,0634$ & 0,0109 & 0,2989 & $-0,4076$ & $-0,0108$ & 0,6788 & $-0,3683$ & 0,0557 & $-0,0998$ & $-0,0801$ & $-0,1188$ & $-0,0042$ & $-0,0571$ \\
\hline
\end{tabular}

${ }^{(1)}$ A importância relativa da característica em cada linha é dada pelo maior coeficiente associado ao menor autovalor $\left(\lambda_{i}\right)$, limitando-se aos autovalores $\left(\lambda_{i}\right)$ menores que 1,0 

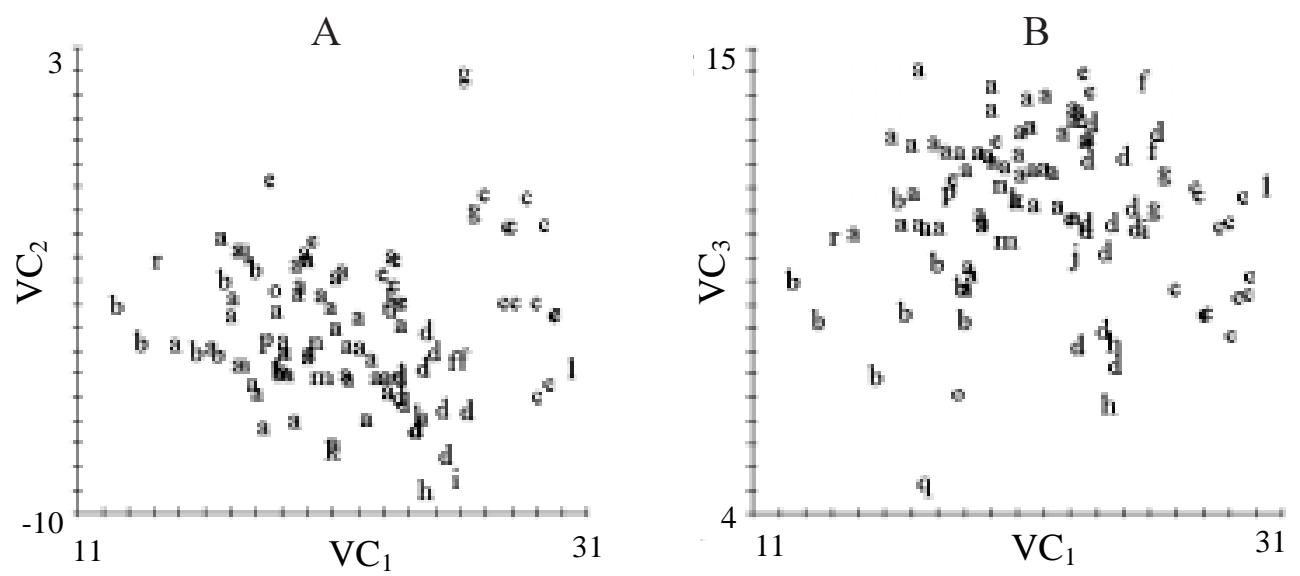

C

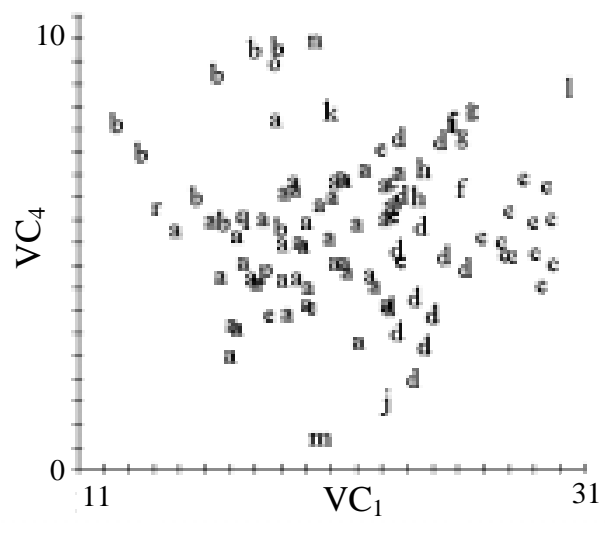

$\mathrm{D}$
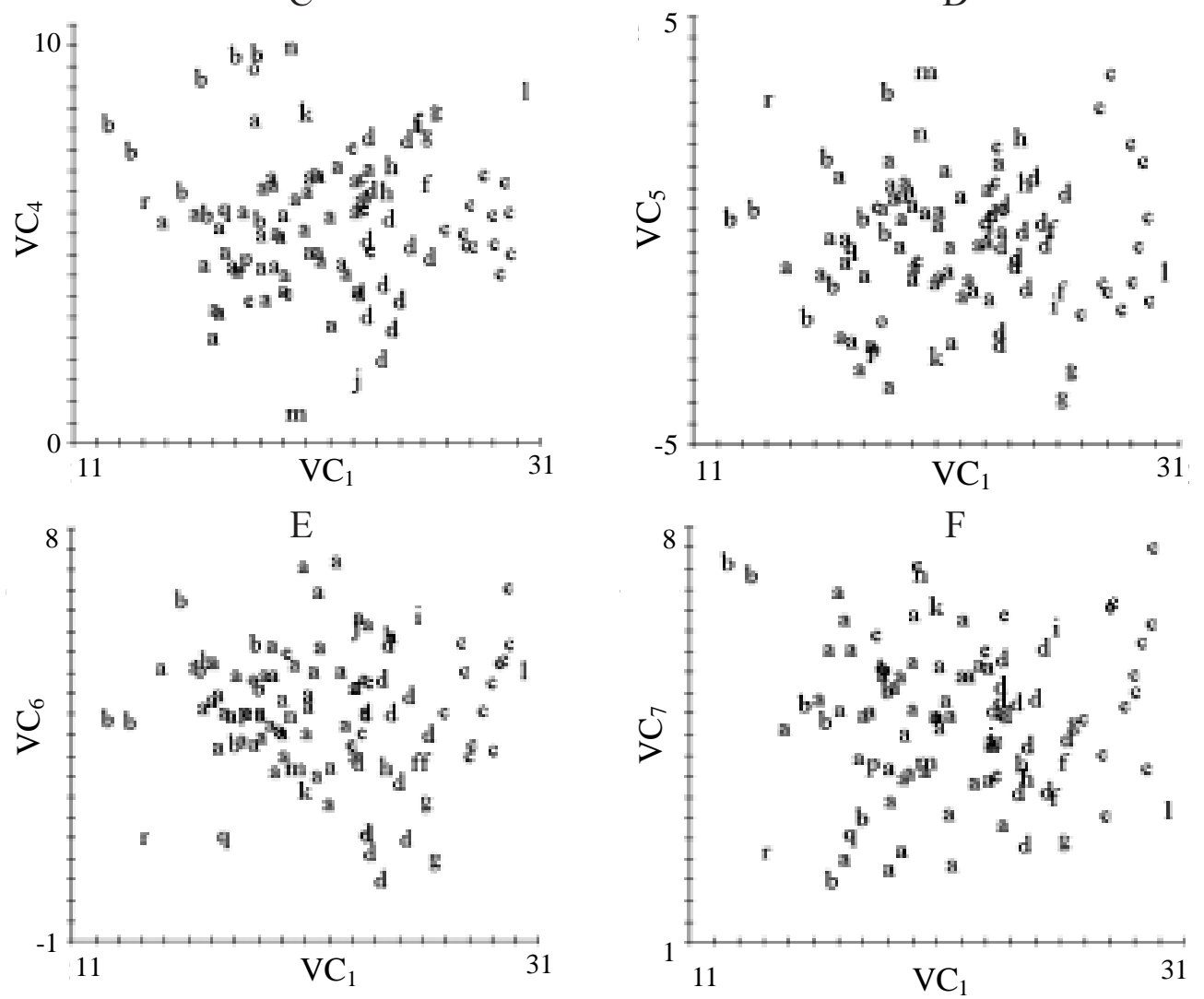

\begin{tabular}{lll}
\hline a: 44 genótipos & b: 8 genótipos & c: 8 genótipos \\
g: 2 genótipos & h: 2 genótipos & i: 1 genótipo \\
m: 1 genótipo & n: 1 genótipo & o: 1 genótipo
\end{tabular}

d: 13 genótipos

e: 6 genótipos

f: 2 genótipos

g: 2 genótipos

n: 1 genótipo

o: 1 genótipo

$\mathrm{j}: 1$ genótipo

$\mathrm{k}$ : 1 genótipo

1: 1 genótipo

Figura 1. Gráficos de dispersão de 99 genótipos de capim-elefante, considerando as variáveis canônicas (VC) 1 e 2 (A), 1 e 3 (B), 1 e 4 (C), 1 e 5 (D), 1 e 6 (E) e 1 e 7 (F). 
pares de progenitores para combinações híbridas, com base no potencial per se dos progenitores (Tabela 1), na magnitude de suas dissimilaridades e na complementação alélica, que é realizada com o interesse de reunir, em uma cultivar, características desejáveis de importância forrageira. Este estudo permitiu identificar genótipos divergentes em relação às testemunhas, proporcionando informações para futuros programas de melhoramento com base em hibridação. Neste caso, recomenda-se seleção nas gerações segregantes, obtidas a partir do cruzamento entre o genitor-elite (testemunhas, preferencialmente o G98, considerado genótipo-padrão) e os que são divergentes, esperando, com isto, o aumento da variabilidade e da possibilidade de identificação de segregantes transgressivos.

Para haver incremento da característica relação folha/colmo, em que foram envolvidas as características comprimento da folha-bandeira, largura da folha-bandeira, comprimento da folha mediana adulta, largura da lâmina na base da folha mediana adulta, largura da lâmina no meio da folha mediana adulta, comprimento da bainha da folha mediana adulta $\mathrm{e}$ comprimento da lígula, relacionadas com a área foliar, são indicados os seguintes cruzamentos: G98 (grupo 5) x G74 (grupo 3) e G98 x G86 (grupo 12). Também são indicados os cruzamentos G98 x G10 (grupo 4), G98 x G53 (grupo 8), G98 x G56 (grupo 17), G98 x

Tabela 4. Importância relativa das 17 e 16 características (após o descarte de diâmetro do colmo), com base em autovetores, com a formação de 18 e 19 grupos de capim-elefante, respectivamente, pelo método de otimização de Tocher, utilizando a distância generalizada de Mahalanobis.

\begin{tabular}{|c|c|c|c|}
\hline \multicolumn{2}{|r|}{ Dezessete características $^{(1)}$} & \multicolumn{2}{|r|}{ Dezesseis características $^{(2)}$} \\
\hline Grupos & Genótipos & Grupos & Genótipos \\
\hline 1 & $\begin{array}{l}72,76,69,51,32,31,30,45,44,96,28, \\
22,92,88,15,83,75,35,78,48,77,34, \\
17,29,27,11,24,91,33,57,64,54,14,9, \\
42,87,1,25,7,70,23,94,21 \text { e } 95\end{array}$ & 1 & $\begin{array}{l}72,76,69,28,92,15,83,22,91,88,78,44, \\
32,35,75,29,24,27,11,77,51,17,48,33, \\
14,9,54,34,57,64,30,69,42,94,95,21, \\
23,93,16,7,70 \text { e } 99\end{array}$ \\
\hline 2 & $40,85,39,84,82,81,67$ e 38 & 2 & $40,85,31,39,84,82,81$ e 67 \\
\hline 3 & $\begin{array}{l}66,74,52,65,61,55,58,68,60,63,89 \text { e } \\
37\end{array}$ & 3 & $66,74,52,65,61,55,58,68,60,63,89$ e 37 \\
\hline 4 & $8,50,5,4,13,12,18,20,43,19,10,59$ e 3 & 4 & $5,8,50,4,13,12,18,20,43,2,19$ e 6 \\
\hline 5 & $26,90,99,93,98$ e 16 & 5 & 26 e 90 \\
\hline 6 & 2 e 6 & 6 & $3,59,62$ e 10 \\
\hline 7 & 47 e 49 & 7 & 80,87 e 1 \\
\hline 8 & 36 e 53 & 8 & 25,38 e 97 \\
\hline 9 & 73 & 9 & 36 e 53 \\
\hline 10 & 62 & 10 & 47 e 49 \\
\hline 11 & 71 & 11 & 98 \\
\hline 12 & 86 & 12 & 86 \\
\hline 13 & 97 & 13 & 71 \\
\hline 14 & 79 & 14 & 41 \\
\hline 15 & 41 & 15 & 79 \\
\hline 16 & 80 & 16 & 73 \\
\hline 17 & 56 & 17 & 56 \\
\hline 18 & 46 & 18 & 45 \\
\hline & & 19 & 46 \\
\hline
\end{tabular}

(1) 1: diâmetro da ráquis; 2: comprimento da folha-bandeira; 3 : largura da folha-bandeira; 4: diâmetro da inflorescência; 5: comprimento da inflorescência; 6: comprimento da lígula; 7: comprimento da arista; 8: comprimento da espigueta; 9: número de flósculos por espigueta; 10: diâmetro do colmo; 11: altura da planta; 12: comprimento da folha mediana adulta; 13: comprimento do entrenó da folha mediana adulta; 14: largura da lâmina na base da folha mediana adulta; 15: largura da lâmina no meio da folha mediana adulta; 16: ângulo de inserção da folha mediana adulta; 17: comprimento da bainha da folha mediana adulta. ${ }^{(2)} 1$ : diâmetro da ráquis; 2 : comprimento da folha-bandeira; 3 : largura da folha-bandeira; 4: diâmetro da inflorescência; 5: comprimento da inflorescência; 6: comprimento da lígula; 7: comprimento da arista; 8: comprimento da espigueta; 9: número de flósculos por espigueta; 10: altura da planta; 11: comprimento da folha mediana adulta; 12: comprimento do entrenó da folha mediana adulta; 13: largura da lâmina na base da folha mediana adulta; 14: largura da lâmina no meio da folha mediana adulta; 15: ângulo de inserção da folha mediana adulta; 16: comprimento da bainha da folha mediana adulta. 
G59 (grupo 4) e G98 x G65 (grupo 3), com base nas características altura da planta, diâmetro do colmo e comprimento do entrenó da folha mediana adulta, que também contribuem para o aumento da relação folha/colmo (Daher, 1993; Shimoya, 2000).

Com base nas características diâmetro e comprimento da inflorescência e número de flósculos por espigueta, que proporcionam maior número de sementes por inflorescência, são indicados os seguintes cruzamentos: G98 (grupo 5) x G6 (grupo 6), G98 x G10 (grupo 4), G98 x G13 (grupo 4), G98 x G18 (grupo 4), G98 x G20 (grupo 4), G98 x G43 (grupo 4), G98 x G50 (grupo 4), G98 x G60 (grupo 3), G98 x G64 (grupo 1) e G98 x G71 (grupo 11). A obtenção de cultivares propagadas por meio de sementes (maior produção e tamanho das sementes) tem sido objetivo do melhoramento do capim-elefante (Diz \& Schank, 1991; Pereira et al., 2001).

A atividade fotossintética e a densidade de plantas por área podem ser alteradas em razão do ângulo de inserção da folha, uma vez que a menor abertura da folha em relação ao caule possibilita melhor captação de luminosidade fotossinteticamente ativa e maior densidade de perfilhos. São indicados os seguintes cruzamentos para melhorar o ângulo de inserção da folha: G98 (grupo 5) x G74 (grupo 3) e G98 x G86 (grupo 12). Também são indicados os cruzamentos G98 x G10 (grupo 4), G98 x G36 (grupo 8), G98 x G56 (grupo 17), G98 x G59 (grupo 4) e G98 x G65 (grupo 3).

\section{Conclusões}

1. A conjugação das técnicas da distância generalizada de Mahalanobis, método de otimização de Tocher, e a análise de variáveis canônicas, possibilitam melhor compreensão das distâncias genéticas relativas entre os genótipos de capim-elefante.

2. O método de otimização de Tocher utilizando a distância generalizada de Mahalanobis permite agrupar os 99 genótipos de capim-elefante pertencentes ao BAGCE, em 18 grupos, e identificar os genótipos G40 e G86 como os mais dissimilares, e o G72 e o G76, como os mais similares.

3. A análise de varáveis canônicas permite visualizar a formação de todos os grupos de genótipos, à medida que se faz a complementação gráfica das variáveis canônicas subseqüentes, até atingir mais de $80 \%$ da variância total.

4. A importância relativa das características que menos contribuem para a divergência genética, com base em autovetores, em ordem de descarte, é: diâmetro do colmo, largura da lâmina no meio da folha mediana adulta, largura da lâmina na base da folha mediana adulta, comprimento da arista, comprimento da espigueta, comprimento da folha mediana adulta, e largura da folha-bandeira.

\section{Referências}

ALI, M.; COPELAND, L. O.; ELIAS, S. G.; KELLY, J. D. Relationship between genetic distance and heterosis for yield and morphological traits in winter canola (Brassica napus L.). Theoretical and Applied Genetics, Berlin, v. 91, n. 1, p. 118-121, 1995.

CARVAlHO, L. P.; CRUZ, C. D.; MORAES, C. F. Genetic divergence in Brazilian cotton, Gossypium hirsutum var. latifolium Hutch. Revista Brasileira de Genética, Ribeirão Preto, v. 18, n. 3, p. 439-443, 1995.

CRUZ, C. D. Aplicação de algumas técnicas multivariadas no melhoramento de plantas. 1990. 188 p. Tese (Doutorado em Genética e Melhoramento de Plantas) - Escola Superior de Agricultura Luiz de Queiroz, Universidade de São Paulo, Piracicaba. 1990.

CRUZ, C. D. Programa GENES; aplicativo computacional em genética e estatística. Viçosa: Editora UFV, 1997. $442 \mathrm{p}$.

CRUZ, C. D.; CARVALHO, S. P.; VENCOVSKY, R. Estudos sobre divergência genética. II. Eficiência da predição do comportamento de híbridos com base na divergência de progenitores. Revista Ceres, Viçosa, v. 41, n. 234, p. 183-190, 1994.

CRUZ, C. D.; REGAZZI, A. J. Modelos biométricos aplicados ao melhoramento genético. 2. ed. Viçosa: UFV, 1997. 390 p.

DAHER, R. F. Caracterização morfológica e isozimática de germoplasma de capim-elefante (Pennisetum purpureum Schum.). 1993. 110 p. Tese (Mestrado em Genética e Melhoramento de Plantas) - Universidade Federal de Viçosa, Viçosa. 1993.

DIZ, D. A.; SCHANK, S. C. Seed and seedling characterization of pearl-millet $\mathrm{x}$ napiergrass hexaploid 
hybrids. Proceedings of the Soil and Crop Science Society of Florida, Belle Grade, v. 50, p. 69-75, 1991

DIZ, D. A.; SCHANK, S. C. Heritabilities, genetic parameters, and response to selection in pearl millet $\mathrm{x}$ elephantgrass hexaploid hybrids. Crop Science, Madison, v. 35, p. 95-101, 1995.

DWIVEDI, A. K.; MITRA, S. K. Divergence analysis of litchi (Litchi chinensis Sonn.) cultivars grown in west Bengal. Indian Journal of Genetics \& Plant Breeding, New Delhi, v. 56, n. 4, p. 486-489, 1996.

JATASRA, D. S.; PARODA, R. S. Genetic divergence in wheat. Indian Journal of Genetics \& Plant Breeding, New Delhi, v. 43, n. 1, p. 63-67, 1983.

JAYLAL; HAIDER, Z. A. Genetic divergence in finger millet (Eleusine coracana). Indian Journal of Agricultural Sciences, New Delhi, v. 64, n. 10, p. $715-716,1994$.

JULFIQUAR, A. W.; VIRMANI, S. S.; CARPENA, A. L. Genetic divergence among some maintainer and restorer lines in relation to hybrid breeding in rice (Oryza sativa L.) Theoretical and Applied Genetics, Berlin, v. 70, n. 6, p. 671-678, 1985.

MIRANDA, J. E. C.; CRUZ, C. D.; COSTA, C. P. Predição do comportamento de híbridos de pimentão (Capsicum annuит L.) pela divergência genética dos progenitores. Revista Brasileira de Genética, Ribeirão Preto, v. 11, n. 4, p. 929-937, 1988.

PANDEY, G.; DOBHAL, V. K. Multivariate analysis in taro (Colocasia esculenta L.). Indian Journal of Genetics \& Plant Breeding, New Delhi, v. 57, n. 3, p. 262-265, 1997.

PEREIRA, A. V.; VALLE, C. B. de; FERREIRA, R. de P.; MILES, J. W. Melhoramento de forrageiras tropicais. In: NASS, L. L.; MELO, I. S. de (Ed.). Melhoramento genético de plantas e de microrganismos. Piracicaba: Fealq, 2001. p. 430-471.
RAO, R. C. Advanced statistical methods in biometric research. New York: John Wiley and Sons, 1952. 390 p.

RAO, A. V.; PRASAD, A. S. R.; KRISHNA, T. S.; SESHU, D. V.; SRINIVASAN, T. E. Genetic divergence among some brown planthopper resistant rice varieties. Indian Journal of Genetics \& Plant Breeding, New Delhi, v. 41, n. 2 , p. 179-185, 1981.

SAMAL, K. M.; JAGADEV, P. N. Genetic divergence among chickpea cultivars. Indian Journal of Genetics \& Plant Breeding, New Delhi, v. 56, n. 1, p. 86-88, 1996.

SANTOS, C. A. F.; MENEZES, E. A.; ARAÚJO, F. P. D. Divergência genética em acessos de guandu. Pesquisa Agropecuária Brasileira, Brasília, v. 29, n. 11, p. $1723-1726,1994$.

SCOTT, A. J.; KNOTT, M. A cluster analysis method for grouping means in the analysis of variance. Biometrics, North Carolina, v. 30, n. 3, p. 507-512, 1974.

SHIMOYA, A. Comportamento per se, divergência genética e repetibilidade em capim-elefante (Pennisetum purpureum Schumacher). 2000. 147 p. Tese (Doutorado em Genética e Melhoramento de Plantas) - Universidade Federal de Viçosa, Viçosa. 2000.

SINGH, Y. P.; KUMAR, A.; CHAUHAN, B. P. S. Genetic divergence in pearl millet. Indian Journal of Genetics \& Plant Breeding, New Delhi, v. 41, n. 2, p. 186-190, 1981.

TCACENCO, F. A. Selection of morphological traits for characterization of elephantgrass accessions. Tropical Grasslands, v. 26, p. 145-155, 1992.

WILCHES, O. M. Evaluación de treinta y cuatro variedades de maní mediante técnicas multivariadas. Revista Instituto Colombiano Agropecuario, Bogotá, v. 18, n. 1, p. 67-76, 1983. 